\title{
Exome sequencing to predict neoantigens in melanoma
}

Antonia L. Pritchard PhD ${ }^{1 *}$, Julie G. Burel B.Sc. ${ }^{2}$, Michelle A. Neller PhD ${ }^{3}$, Nicholas K. Hayward PhD

${ }^{1}$, J. Alejandro Lopez $\mathrm{MD}^{3,4}$, Martina Fatho B.Sc ${ }^{5}$, Volker Lennerz PhD ${ }^{5}$, Thomas Wölfel MD ${ }^{5}$, Christopher W. Schmidt PhD ${ }^{3}$

1. Oncogenomics, Level 8 CBCRC building, QIMR Berghofer Medical Research Institute, 300 Herston Road, Herston, Brisbane, QLD, 4006, Australia

2. Molecular Vaccinology, Level 12 Central building, QIMR Berghofer Medical Research Institute, 300 Herston Road, Herston, Brisbane, QLD, 4006, Australia

3. Cancer Immunotherapy, Level 10 CBCRC building, QIMR Berghofer Medical Research Institute, 300 Herston Road, Herston, Brisbane, QLD, 4006, Australia

4. School of Natural Sciences, Griffith University, Kessels Road, Brisbane, QLD, 4111, Australia

5. Third Medical Clinic (Hematology/Oncology/Pneumology), University Medical Center, Johannes Gutenberg University, Mainz, Germany

* Corresponding author: Antonia L. Pritchard:

Oncogenomics, Level 8, CBCRC Building, QIMR Berghofer Medical Research Institute, 300 Herston Road, Herston, Brisbane, QLD, 4006, Australia

Phone Number: +61733620323 Fax Number: +61 738453508

Email: Antonia.Pritchard@qimrberghofer.edu.au

Sources of funding: AP: Cure Cancer Australia; AP, CS, NH: Rio Tinto Ride to Conquer Cancer; AP, NH: Buck-Off Melanoma; CS, NH: National Health and Medical Research Council

Conflicts of interest: None to declare

Word count:

Abstract word count: 192

Article word count: 1701

Brief title: Exome sequencing to predict melanoma neoantigens

Keywords: Neoantigen, epitope prediction, MHC class I, cytotoxic T cells, melanoma, cancer, immunotherapy, clinical immunology 


\begin{abstract}
The ability to use circulating peripheral blood cells and matched tumour sequencing data as a basis for neoantigen prediction has exciting possibilities for application in the personalised treatment of cancer patients. We have used a high throughput screening approach, combining whole-exome sequence data, mRNA microarrays and publically available epitope prediction algorithm output to identify mutated proteins processed and displayed by patient tumours and recognised by circulating immune cells. Matched autologous melanoma cell lines and peripheral blood mononuclear cells were used to create mixed lymphocyte tumour cell cultures (MLTC), resulting in an expansion of tumour-reactive T cells to use for mutated peptide screening. Five patients were investigated, three of whom had a durable complete response (CR; $15+$ years) in an autologous melanoma-pulsed dendritic cell clinical trial. We identified seven mutated antigens in total that stimulated T-effector memory cells in two of the five patients. While the procedure did not result in clinically applicable neoantigens for all patients, those identified were likely important in tumour clearance, leading to durable CR. The nature of the screening process allows results to be obtained rapidly and is easily applicable to a wide variety of different tumour types.
\end{abstract}




\section{Introduction}

While significant advances in therapeutic options for malignant melanoma have been made in recent years, when it has metastasised it still kills $>85 \%$ of patients within five years (1). Common tumour antigen-based immunotherapies can potentially selectively target cancer cells; however, this form of immunotherapy has yet to yield consistent meaningful clinical outcomes (2). It has been hypothesised that patient-specific neoepitopes, derived from protein-altering mutational events, result in stronger immune responses against the expressing tumour cells than common tumour antigens (3). Given the person-specific nature of these neoepitopes, they have been traditionally hard to identify using labour intensive 'forward' immunology approaches. Additionally, these methods are unsuitable for timely epitope identification for patients in a clinical setting, in which the median survival time of stage IV melanoma patients is only $6-9$ months.

Application of a rapid 'reverse' methodology has been used to successfully predict immunogenic neoepitopes from tumour exome sequencing data recognised by tumour-infiltrating lymphocytes (TIL) $(4,5)$. It is not always possible to isolate/obtain TILs from tumours, so we have established a methodology for identification of neoepitopes using circulating peripheral blood mononuclear cells (PBMC). Here, we demonstrate the application of a 'reverse' immunology approach using tumour cell lines established from metastases of five stage IV melanoma patients $(6,7)$ and recognised by T cells derived from PBMCs and expanded using mixed lymphocyte tumour culture (MLTC).

\section{Materials and Methods}

Tumour-specific mutations were identified using whole exome sequencing, as previously described (8). Briefly, the matched blood and tumour pairs for samples A02, A06, D05 and D14 were sequenced using Illumina technology, while D41 was sequencing using the SOLiD platform. The samples were aligned to the hg19 reference genome and all variants were called using ANNOVAR (9) and those unique to the tumour samples were identified. The minimum read depth was $\geq$ 10 reads and the minimum mutation call had to be $\geq 20 \%$ of all reads; using Illumina OmniQuad arrays, the false negative rate was determined to be $0.45 \%$ and through Sanger sequencing of variants, the false positive rate was determined to be $2 \%$. The cell lines used in this work were created as part of the clinical trial protocols for a dendritic cell (DC) vaccine; they were created from metastatic lesions. The cell lines were tested against germline DNA from the blood to ensure they were correctly matched, by using STR profiling of 10 markers (QIMR Berghofer Scientific Services department); cell lines were additionally ensured they matched each other by whole exome sequencing.

Once the non-synonymous and frameshift mutations were identified (total $n=1061$; Supplementary Table 1), they were verified by Sanger sequencing. Whether the gene was expressed or not was assessed using mRNA microarray (HT12v4, Illumina) and those that were undetectable were removed from further assessment. For each confirmed mutation in expressed genes, the 11 amino acids either side of the mutation were obtained using a LINUX-based script (available on request) based on the CDNA positional information output from Annovar annotation of variants. Frameshift mutations were manually curated. Identification of 9- and 10-mer peptides predicted to bind to patient-specific HLA-A and -B subtypes were carried out using this 21-mer and five programs employing different algorithms: SYFPEITHI (support vector machine modelling) (10), RANKPEP (matrix based method) (11), ANN (artificial neural network) (12), SMM (stabilized matrix method) (13) and NetMHCpan 2.8 (an ANN, based on primate, mouse, cattle and pig training sets) (14); a summary of the total data is in Supplementary Figure 1; raw data are in Supplementary Table 2. HLA-C predictions were not employed, due to current limitations in predictive power (15).

MLTC were created using a standard protocol (3) and $70-88 \%$ of the final culture were CD8 ${ }^{+}$(Supplementary Table 3). IFN $\gamma$ ELISpot was performed using peptides at $2 \mu \mathrm{g} / \mathrm{mL}$ to stimulate MLTC cultured in AIM-V media, 10IU/mL IL2 and 10\%

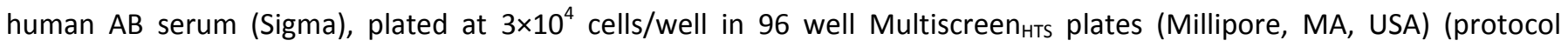
available on request; antibodies used are detailed in Supplementary Table 4). Intracellular cytokine staining (ICS) was performed (antibodies used are detailed in Supplementary Table 4) on an LSR-Fortessa (BD Biosciences) and analysed using FlowJo (FlowJo LLC, USA). Positive controls were autologous tumour and PHA (ELISpot) and PMA/ionomycin (ICS); unstimulated wells allowed assessment of background MLTC stimulation. 
Wildtype and mutant DNMT1 ORF (position 2086 to 2707) was amplified in a nested PCR from the D14 melanoma cell line (Forward GCCATGAAGGAGGCAGATGACG; Reverse CCTCATCTCAGCCAGACGGG), and purified PCR products were cloned into pcDNATM3.1/V5-his TOPO ${ }^{\circledR}$ TA (Life Technologies, Germany) and sequenced to identify a wildtype and mutant construct. COS-7 cells $\left(2 \times 10^{4}\right)$ were transiently co-transfected (Lipofectamine 2000, Life Technologies) with HLA-A*24:02cDNA or HLA-B*07:02 (100ng) and either mutant or wt-DNMT1 plasmids (300 ng) (with a control of HLA-A*24:02-cDNA or HLA-B*07:02 only) on MultiScreen-IP HTS plates (Merck Millipore, Germany) coated with anti-IFN $\gamma$ monoclonal antibody (Mab1-D1K, $10 \mu \mathrm{g} / \mathrm{ml}$; Mabtech, Sweden). After 24h, 5000 or 1000 cells of D14-CTL clone 1B/85 were added and incubated for $20 \mathrm{~h}$ before IFN $\gamma$ ELISpot assays were performed. Additional controls were unstimulated CTLs and CTLS stimulated with D14-MEL\#7 and D14-LCL (both at 50,000 cells/well).

\section{Results and discussion}

One of the major obstacles to using patient-specific neoepitopes in tumour immunotherapy is the timely identification of immunogenic peptide sequences. Recent technologic advances allow the coding mutations in tumours to be relatively easily and rapidly identified and have been used to successfully predict the neoepitopes recognised by TILs $(4,5)$; unfortunately, it not always possible to isolate or obtain TILs. We have therefore developed a method to identify immunogenic neoepitopes using peripheral circulating lymphocytes, which does not rely on a strong ex vivo response for reactivity to be detected. The use of MLTC increases the likelihood of detection of rare reactive T cells, due to proliferation of tumour-reactive $\mathrm{CD} 8^{+} \mathrm{T}$ cells, and peripheral blood is easy to obtain from all patients.

Three of the patients assessed, D05, D14 and D41, experienced long-lasting durable complete response (CR) to DC immunotherapy of greater than 15 years, to date $(6,7)$. PBMCs from all five patients had $\mathrm{CD}^{+} \mathrm{CD}^{+} \mathrm{IFN} \gamma^{+} \mathrm{T}$-cell expansion in response to co-culture with autologous tumour (Supplementary Table 3).

After Sanger sequencing verification of non-synonymous and frameshift mutations identified by whole-exome sequencing (Supplementary Table 1), followed by mRNA microarray confirmation of gene expression and acquisition of the 11 amino acids on either side of the mutation, the 21-mer amino acid sequences were screened through five MHC-class I epitope prediction programs (10-14). Comparison between the algorithms revealed substantial differences in the number of epitopes predicted by individual mutation; SMM consistently predicted epitopes for a higher number of mutations, while SYFPEITHI and RANKPEP predicted epitopes for a fewer number (Supplementary Figure 1). ANN, SMM and NetMHCpan have an analogous scoring system allowing comparison between the resultant predicted epitopes. SMM predicted weaker binding affinities both when individual cell lines and the HLA-types in common between multiple cell lines (HLA$A * 02, H L A-A * 03$ and HLA-B*27) were assessed (Figure 1). These data therefore support the recent hypothesis that these algorithms have distinct binding affinities for different HLA subtypes, with HLA-A*02 having a greater proportion of traditionally classified 'strong binders' predicted than HLA-A*03 and HLA-B*27 (16). In addition, the recent work by Duan and colleagues (17) also suggested caution in using the traditional binding categories to assess results from the epitope prediction programs. This work demonstrated that mutations in an anchor residue for class-I MHC binding that resulted in a more rigid peptide were able to elicit a $\mathrm{CD}^{+}{ }^{+} \mathrm{T}$-cell response, regardless of the predicted binding affinity $\mathrm{K}^{\mathrm{d}}$ score. It is therefore clear that while these epitope prediction algorithms are useful tools, there are important caveats to their use that must be considered and future improvements are necessary before they can reliably predict the majority of immunogenic peptides present.

Results from the epitope prediction analyses were ranked based on binding scores from each model, with ANN, SMM or NetMHCpan scores $\leq 500 \mathrm{nM}$ plus support from at least one other method selected for peptide synthesis (Pepsets; Mimotopes. Total dataset: Supplementary Table 2). The individual total number of mutations and complexity of HLA genotypes reflected the number of potential peptides that ranked within the cut-off criteria for testing (Supplementary Table 1).

In total, 642 peptides were tested in triplicate by ELISpot (raw data: Supplementary Figure 2). Peptides stimulating positive ELISpot response ( $>30$ spots once background was subtracted) were re-tested by ELISpot and by ICS. Five peptides consistently elicited IFN $\gamma$ responses from the MLTC, as assessed by both ELISpot and ICS and two responses were detectable by ELISpot alone (Table 1). Comparison of the algorithms predicting epitope binding for these seven peptides

Downloaded from cancerimmunolres.aacrjournals.org on June 11, 2015. @ 2015 American Association for Cancer Research. 
revealed that only NetMHCpan predicted all seven would bind (Table 1). Responses were particularly strong against mutated peptides derived from CCT6A (D05) and DMNT1 (D14). CCT6A and WASL neoepitopes were also identified via a 'forward' cDNA expression and cloning approach ((3) and Lennerz and colleagues, manuscript in preparation). Additionally, clear populations of IFN $\gamma^{\text {high }}$ cells were observed (Table 1; FACS plots in Supplementary Figure 3). Given the strength of response, the DMNT1 neoepitope from patient D14 (IYKAPCENW) was selected to confirm the predicted HLA$A * 24: 02$ restriction and that internal protein processing resulted in the predicted neoepitope (Figure 2). CCT6A (patient D05) was previously confirmed to be restricted through HLA-B*27:05, but WASL (patient D14) was found to be restricted through HLA-B*51:01 (Lennerz and colleagues, manuscript in preparation), where it was predicted by the algorithms to bind HLA-B*07:02 (e.g. by ANN: $B * 51: 01=1379 n M$ vs $B * 07: 02=66 n M)$.

Taken together (Table 1; Figure 1; Supplementary Figure 1), these data indicate that while this approach was able to successfully identify and assign HLA restriction of neoepitopes stimulating a strong immune response, there is also a serious potential for false-negative results, depending on the choice of algorithm and interpretation of results.

Intriguingly, upon further scrutiny of PABPC3 (patient D14) it was revealed that the dinucleotide mutation (GA/AG) resulted in a 9-mer peptide sequence that is $100 \%$ homologous to gene family member PABPC1 (whole-exome sequencing alignment: Supplementary Figure 4). While PABPC1 is ubiquitously expressed, PABPC3 has been reported as being testis-specific (18); mRNA microarray analysis showed that both genes were expressed by the D14 melanoma cell line. The reason that tolerance to this peptide was not induced is therefore intriguing and suggests that mutations resulting in endogenous sequences may still have immunogenic potential.

Mid-expansion MLTC (day 18) were assessed for responses to tumour/peptides. Despite fewer total CD8 ${ }^{+}$cells, the proportion of IFN $\gamma^{+}$cells within this population was higher at day 18 than at day 34 (Figure 3) and responses were detectable by ELISpot and ICS, suggesting MLTC generation could be expedited for clinical application, while still retaining advantages over the use of ex vivo cells. The $\mathrm{CD}^{+}{ }^{+} \mathrm{CD} 8^{+} \mathrm{IFN} \gamma^{+}$cells were phenotyped using memory T-cell markers (CCR7, CD27 and CD45RA), revealing that the majority were of an effector memory phenotype (Figure 3; Gating strategy: Supplementary Figure 5). The populations of naive antigen-specific T cells declined between days 18 and 34, suggesting differentiation occurred during MLTC expansion (Figure 3).

Intriguingly, no consistently positive results were identified for A02, A06 or D41 by ELISpot or ICS, despite MLTC reactivity to autologous tumour (Table 1). Notably, D41 had durable CR resulting from DC immunotherapy (7), indicating that their immune response to autologous tumour was clinically effective. It is possible that these patients do not react to any neoepitopes and indeed D41 responds to three HLA-A-restricted 'traditional' cancer epitopes (data not shown); A02 and A06 are untested. The prediction algorithms or filtering process employed may have removed viable immunogenic neoepitopes, T-cell responses due to other mechanisms of creating neoepitopes (such as unidentified frameshifts, in/dels, or chromosomal translocations/gene fusions), or neoepitopes restricted through HLA-C/MHC-class II might be important in these patients. Having used sequencing data from tumour cell lines, it is possible that a non-endogenous mutational artefact developed in vitro may have been tested in this study, or that a chromosomal aberration event may have occurred with culturing, masking the presence of an important endogenous mutation. It is also possible that immunogenic clonal mutations present in the tumour mass, but not grown out in the cell line, may not have been detected.

The patient samples used in this study are from clinical trials conducted over 15 years ago and as a result TILs are not available to perform a comparison with the reactivity identified in circulating $\mathrm{CD} 8^{+} \mathrm{T}$ cells. Given the recently published methods for identification of neoepitopes on TILs $(4,5)$, such investigations are now possible and would provide vital information on the distribution and diversity of neoepitope-reactive T cells within the body. Additionally, combining assessments of the neoepitope landscape in TILs and circulating T-cell neoepitopes in patients with differing clinical responses to immunotherapies is likely to provide illuminating information, as recently suggested for clinical response to CTLA-4 (19).

In conclusion, while we have shown the current analysis procedures do not result in clinically applicable neoepitopes for all patients, for D05 and D14, the identified neoepitopes were potentially important in their durable CR due to DC 
Author Manuscript Published OnlineFirst on June 5, 2015; DOI: 10.1158/2326-6066.CIR-15-0088

Author manuscripts have been peer reviewed and accepted for publication but have not yet been edited.

immunotherapy (6). This new methodology in cancer epitope prediction therefore has exciting possibilities for application in the personalised treatment of certain patients. 


\section{Acknowledgements:}

ALP is supported by Cure Cancer Australia (APP1051048). CS is supported by the Rio Tinto Ride to Conquer Cancer (QIMR Berghofer Flagship award) and has ongoing support from the Alpha Delta chapter of Phi Beta Sigma. NH is supported by the NHMRC (APP1026112). This work was funded by Cure Cancer Australia (APP1051048), the Rio Tinto Ride to Conquer Cancer (QIMR Berghofer Flagship award) and Buck Off Melanoma. The authors would like to thank Mitchell Stark, Peter Johansson and Lutz Krause for computational support. We are grateful to the patients who donated samples, allowing this research to be carried out. 


\section{References:}

1. Dickson PV, Gershenwald JE. Staging and prognosis of cutaneous melanoma. Surg Oncol Clin N Am. 2011;20:1-17.

2. Neller MA, Lopez JA, Schmidt CW. Antigens for cancer immunotherapy. Semin Immunol. 2008;20:286-95.

3. Lennerz V, Fatho M, Gentilini C, Frye RA, Lifke A, Ferel D, et al. The response of autologous T cells to a human melanoma is dominated by mutated neoantigens. Proc Natl Acad Sci U S A. 2005;102:16013-8.

4. Robbins PF, Lu YC, El-Gamil M, Li YF, Gross C, Gartner J, et al. Mining exomic sequencing data to identify mutated antigens recognized by adoptively transferred tumor-reactive T cells. Nat Med. 2013;19:747-52.

5. Yadav M, Jhunjhunwala S, Phung QT, Lupardus $P$, Tanguay J, Bumbaca S, et al. Predicting immunogenic tumour mutations by combining mass spectrometry and exome sequencing. Nature. 2014;515:572-6.

6. O'Rourke MG, Johnson M, Lanagan C, See J, Yang J, Bell JR, et al. Durable complete clinical responses in a phase I/II trial using an autologous melanoma cell/dendritic cell vaccine. Cancer Immunol Immunother. 2003;52:38795.

7. O'Rourke MG, Johnson MK, Lanagan CM, See JL, O'Connor LE, Slater GJ, et al. Dendritic cell immunotherapy for stage IV melanoma. Melanoma Res. 2007;17:316-22.

8. Stark MS, Woods SL, Gartside MG, Bonazzi VF, Dutton-Regester K, Aoude LG, et al. Frequent somatic mutations in MAP3K5 and MAP3K9 in metastatic melanoma identified by exome sequencing. Nat Genet. 2012;44:165-9.

9. Wang K, Li M, Hakonarson H. ANNOVAR: functional annotation of genetic variants from high-throughput sequencing data. Nucleic Acids Res. 2010;38:e164.

10. Rammensee H, Bachmann J, Emmerich NP, Bachor OA, Stevanovic S. SYFPEITHI: database for MHC ligands and peptide motifs. Immunogenetics. 1999;50:213-9.

11. Reche PA, Reinherz EL. Prediction of peptide-MHC binding using profiles. Methods Mol Biol. 2007;409:185200.

12. Nielsen M, Lundegaard C, Worning P, Lauemoller SL, Lamberth K, Buus S, et al. Reliable prediction of T-cell epitopes using neural networks with novel sequence representations. Protein Sci. 2003;12:1007-17.

13. Peters B, Sette A. Generating quantitative models describing the sequence specificity of biological processes with the stabilized matrix method. BMC Bioinformatics. 2005;6:132.

14. Hoof I, Peters B, Sidney J, Pedersen LE, Sette A, Lund O, et al. NetMHCpan, a method for MHC class I binding prediction beyond humans. Immunogenetics. 2009;61:1-13.

15. Rasmussen M, Harndahl M, Stryhn A, Boucherma R, Nielsen LL, Lemonnier FA, et al. Uncovering the peptidebinding specificities of HLA-C: a general strategy to determine the specificity of any MHC class I molecule. J Immunol. 2014;193:4790-802.

16. Paul S, Weiskopf D, Angelo MA, Sidney J, Peters B, Sette A. HLA class I alleles are associated with peptidebinding repertoires of different size, affinity, and immunogenicity. J Immunol. 2013;191:5831-9.

17. Duan F, Duitama J, Al Seesi S, Ayres CM, Corcelli SA, Pawashe AP, et al. Genomic and bioinformatic profiling of mutational neoepitopes reveals new rules to predict anticancer immunogenicity. J Exp Med. 2014;211:2231-48.

18. Feral C, Guellaen G, Pawlak A. Human testis expresses a specific poly(A)-binding protein. Nucleic Acids Res. 2001;29:1872-83.

19. Snyder A, Makarov V, Merghoub T, Yuan J, Zaretsky JM, Desrichard A, et al. Genetic basis for clinical response to CTLA-4 blockade in melanoma. N Engl J Med. 2014;371:2189-99. 
Table 1: Peptides eliciting IFN $\gamma$ release from MLTC measured by ELISpot and intracellular FACS.

The predicted HLA-restriction, antigen used to stimulate response, ELISpot well and the percentage of IFN $\gamma$-producing $\mathrm{CD}^{+}$, and $\mathrm{CD}^{+}$IFN $\gamma^{\text {high }}$ cells present in MLTC are shown; the percentages detected in unstimulated samples are shown, and were not subtracted from stimulated values. The percentage of each lineage in the total MLTC population is indicated for each cell line in the shaded grey section.

\begin{tabular}{|c|c|c|c|c|c|c|c|}
\hline $\begin{array}{l}\text { Patient } \\
\text { ID }\end{array}$ & Antigen & $\begin{array}{l}\text { Predicted } \\
\text { HLA } \\
\text { restriction }\end{array}$ & $\begin{array}{l}\text { Algorithms } \\
\text { predicting HLA } \\
\text { binding }\end{array}$ & $\begin{array}{l}\text { Positive } \\
\text { Detection } \\
\text { Method }\end{array}$ & ELISpot & $\begin{array}{l}\mathrm{CD3}^{+} \mathrm{CD}^{+} \\
\text {ICS }\end{array}$ & $\begin{array}{c}\text { IFN } \gamma^{\text {high }} \\
\mathrm{CD}^{+} \\
\mathrm{CD}^{+}{ }^{+} \text {ICS }\end{array}$ \\
\hline D05 & - & - & & & - & $85 \%$ & $\mathrm{n} / \mathrm{a}$ \\
\hline D05 & Unstimulated & - & - & - & & $0.137 \%$ & $0.01 \%$ \\
\hline D05 & Tumour & - & - & $\begin{array}{l}\text { ELISpot } \\
\text { and ICS }\end{array}$ & & $8.71 \%$ & $1.16 \%$ \\
\hline D05 & CCT6A: LRTKV $\underline{Y} A E L$ & $B * 27: 05$ & $\begin{array}{l}\text { ANN, SMM, } \\
\text { NetPan }\end{array}$ & $\begin{array}{l}\text { ELISpot } \\
\text { and ICS }\end{array}$ & & $33.60 \%$ & $5.57 \%$ \\
\hline D05 & TRRAP: LLYQELLLPL & $A * 02: 01$ & All & ELISpot & & $0.22 \%$ & $0.01 \%$ \\
\hline D14 & - & - & & & - & $87 \%$ & $n / a$ \\
\hline D14 & Unstimulated & - & & - & & $1.75 \%$ & $0.18 \%$ \\
\hline D14 & Tumour & - & & $\begin{array}{l}\text { ELISpot } \\
\text { and ICS }\end{array}$ & & $17.50 \%$ & $1.55 \%$ \\
\hline D14 & DNMT1: IYKAPCEENW & $A * 24: 02$ & $\begin{array}{l}\text { Rankpep, } \\
\text { SMM, Netpan }\end{array}$ & $\begin{array}{l}\text { ELISpot } \\
\text { and ICS }\end{array}$ & & $38.90 \%$ & $6.36 \%$ \\
\hline D14 & PABPC3: YYPPSQIAQ & $A * 24: 02$ & $\begin{array}{l}\text { SYFP, ANN, } \\
\text { SMM, Netpan }\end{array}$ & $\begin{array}{l}\text { ELISpot } \\
\text { and ICS }\end{array}$ & & $10.60 \%$ & $2.17 \%$ \\
\hline D14 & MAGEA10: LYNGMEHLI & $A * 24: 02$ & $\begin{array}{l}\text { Rankpep, ANN, } \\
\text { SMM, Netpan }\end{array}$ & $\begin{array}{l}\text { ELISpot } \\
\text { and ICS }\end{array}$ & & $15.60 \%$ & $3.95 \%$ \\
\hline D14 & FMN2: HSVSSAFK吕 & $A * 0301$ & $\begin{array}{l}\text { ANN, SMM, } \\
\text { Netpan }\end{array}$ & $\begin{array}{l}\text { ELISpot } \\
\text { and ICS }\end{array}$ & & $29.00 \%$ & $7.45 \%$ \\
\hline D14 & WASL: YPPPPPALL & $B * 07: 02$ & $\begin{array}{l}\text { Rankpep, ANN, } \\
\text { SMM, Netpan }\end{array}$ & ELISpot & & $0.72 \%$ & $0.15 \%$ \\
\hline D41 & - & - & & & - & $85 \%$ & $n / a$ \\
\hline D41 & Unstimulated & - & & - & & $0.04 \%$ & $0.00 \%$ \\
\hline D41 & Tumour & - & & $\begin{array}{l}\text { ELISpot } \\
\text { and ICS }\end{array}$ & & $8.85 \%$ & $1.07 \%$ \\
\hline $\mathrm{A} 02$ & - & - & & & - & $80 \%$ & $n / a$ \\
\hline $\mathrm{A} 02$ & Unstimulated & - & & - & & $0.47 \%$ & $0.09 \%$ \\
\hline A02 & Tumour & - & & $\begin{array}{l}\text { ELISpot } \\
\text { and ICS }\end{array}$ & & $18.40 \%$ & $4.47 \%$ \\
\hline A06* & - & - & & & - & $20 \%$ & $n / a$ \\
\hline A06* & Unstimulated & - & & - & & $2.49 \%$ & $0.17 \%$ \\
\hline A06* & Tumour & - & & $\begin{array}{l}\text { ELISpot } \\
\text { and ICS }\end{array}$ & & $29.60 \%$ & $9.79 \%$ \\
\hline
\end{tabular}

* ELISpot performed on cells selected on the basis of CD8 by autoMACS (Miltenyi) at day 28 and further cultured with irradiated tumour for 10 days, making a total of 44 days; insufficient cells remained for ICS, so this was performed on day 18 cells alone. 
Figure 1: Three prediction algorithms (ANN, SMM and NetMHCpan) have analogous scoring mechanisms, allowing comparison. For each individual patient, the cumulative percentage of each score, from strong binding $(<50 \mathrm{nM})$ up to the weaker binding scores $(<500 \mathrm{nM})$ are plotted (graphs A-E), allowing overall comparison of the strength of predictions between algorithms. As patients shared some HLA types, the cumulative percentage of each score from strong binding $(<50 \mathrm{nM})$ up to the weaker binding scores $(<500 \mathrm{nM})$ are plotted, allowing overall comparison of the strength of HLA specific predictions between algorithms. The numbers of patients' data making up each graph are as follows: HLA-A*02, $n=4 ;$ HLA-A*03, $n=2$; and HLA-B*27, $n=3$.

Figure 2: Investigation of reactivity of D14 HLA-A*24:02 cytotoxic lymphocyte (CTL) clones against DMNT1 mutated and wild type peptides

(A) K562 cells transfected with HLA-A*24:02 CDNA and pulsed with DNMT1 wild type and mutated peptides (at $5 \mu \mathrm{M})$ were tested against D14 HLA-A*24:02 specific CTL clones (5,000/well)

(B) To assess the internal processing of the DMNT1 mutated and wild type protein, TOPO-TA-cloning of the mutated and wild type DMNT1 CDNA sequences from D14 into expression vector pcDNA3.1/V5 HIS TOPO was carried out. Testing was performed against COS-7 cells transfected with HLA-A*24 or HLA-B*07, in order to show the specificity of the DMNT1 mutant clone.

Figure 3: Comparison of MLTC responses to tumour/ peptide stimuli at day 18 and day 34 of growth.

The total percentage of $\mathrm{CD}^{+} \mathrm{CD} 8^{+} \mathrm{T}$ cells present in the MLTC culture and the proportion of those that elicit IFN $\gamma$ in response to peptide/tumour are shown above the bar chart. The graph illustrates the proportion of cells expressing T-cell memory phenotype markers CD45RA, CCR7 and CD27 in $\mathrm{CD}^{+} \mathrm{CD}^{+} \mathrm{IFN} \gamma^{+}$cells. 
Figure 1

D05 (HLA-A*02, -B*27, -B*44)
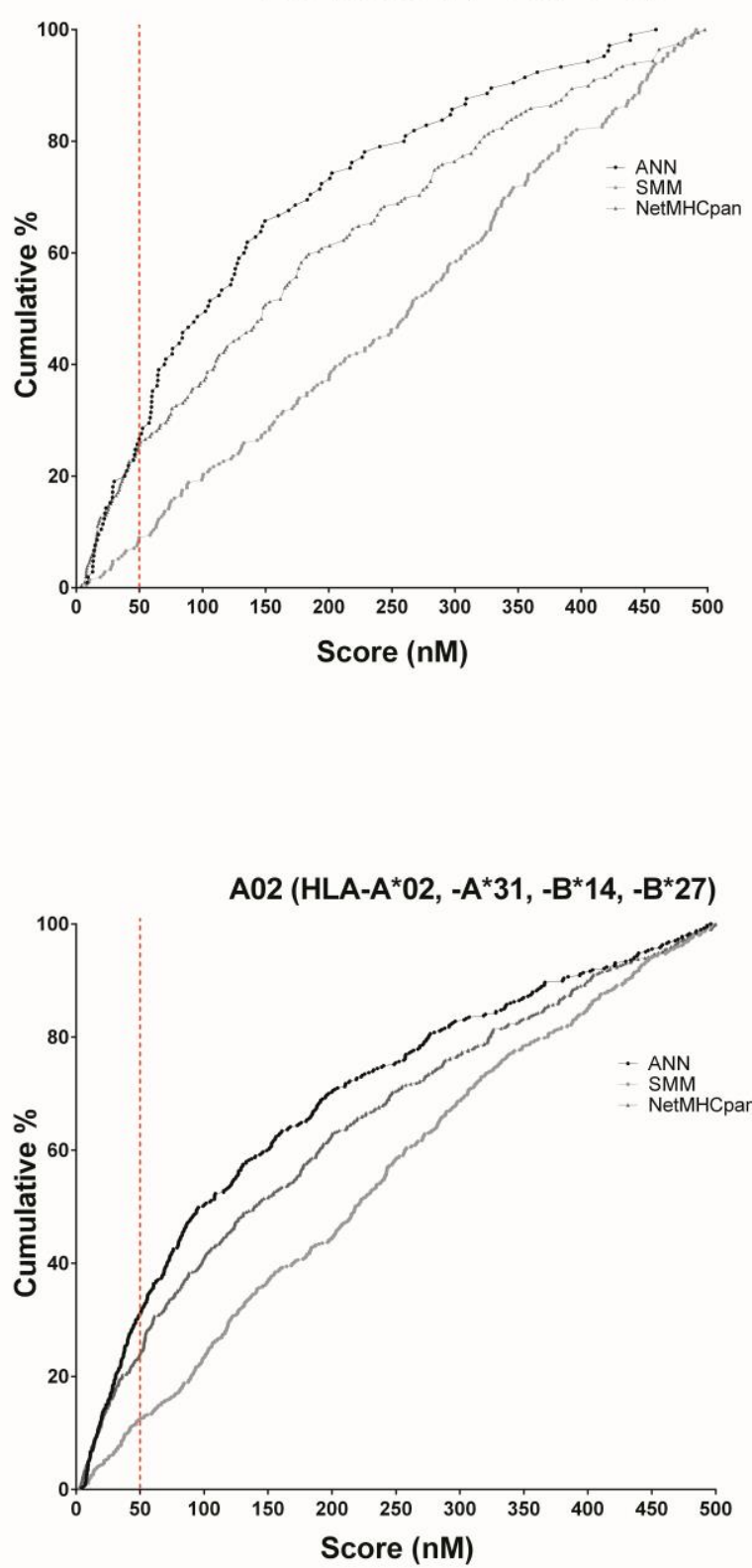

D14 (HLA-A*03, -A*24, - $\left.B^{*} 07\right)$
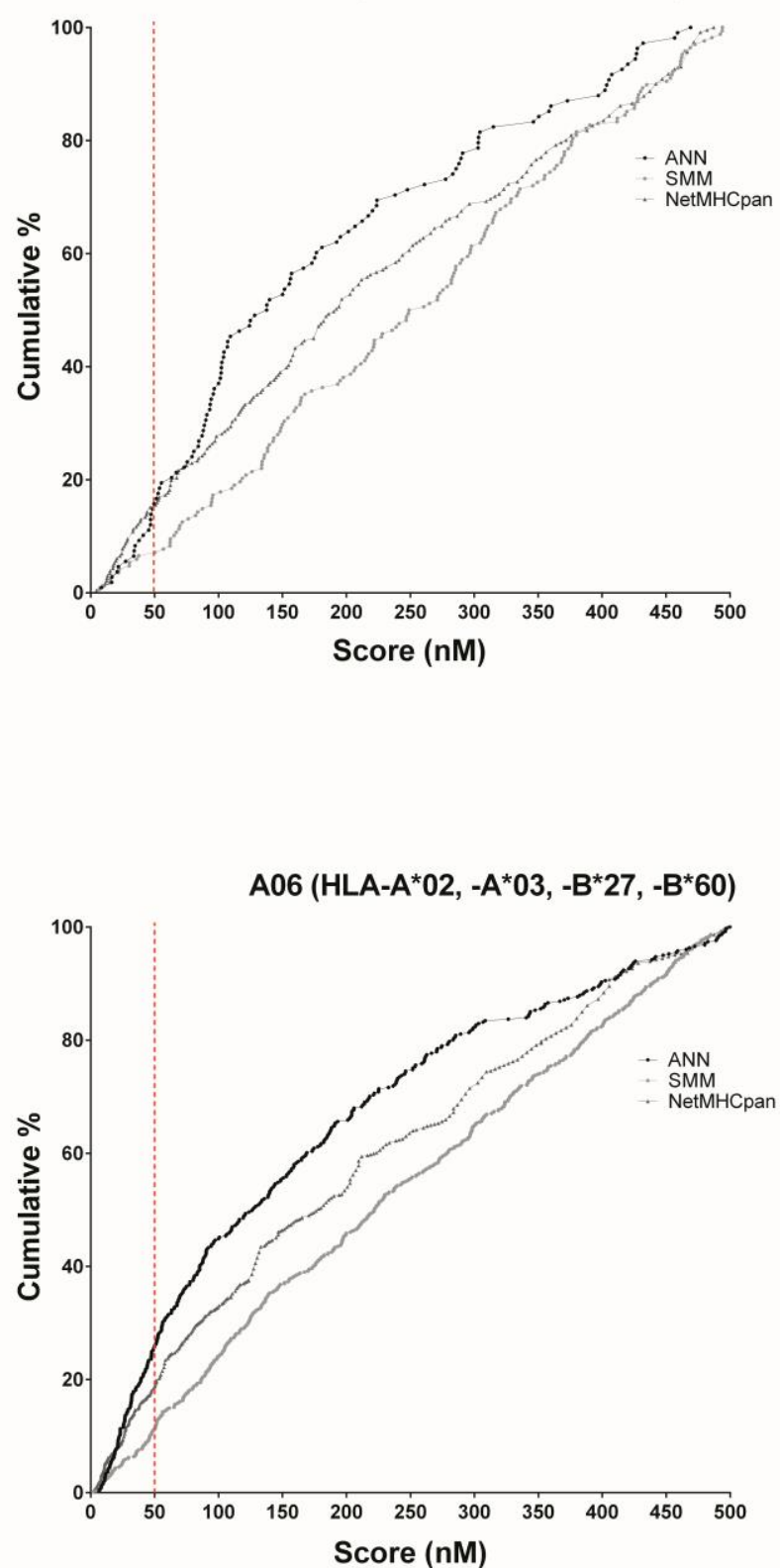

D41 (HLA-A*01, - A $\left.^{*} 02,-B^{*} 08\right)$
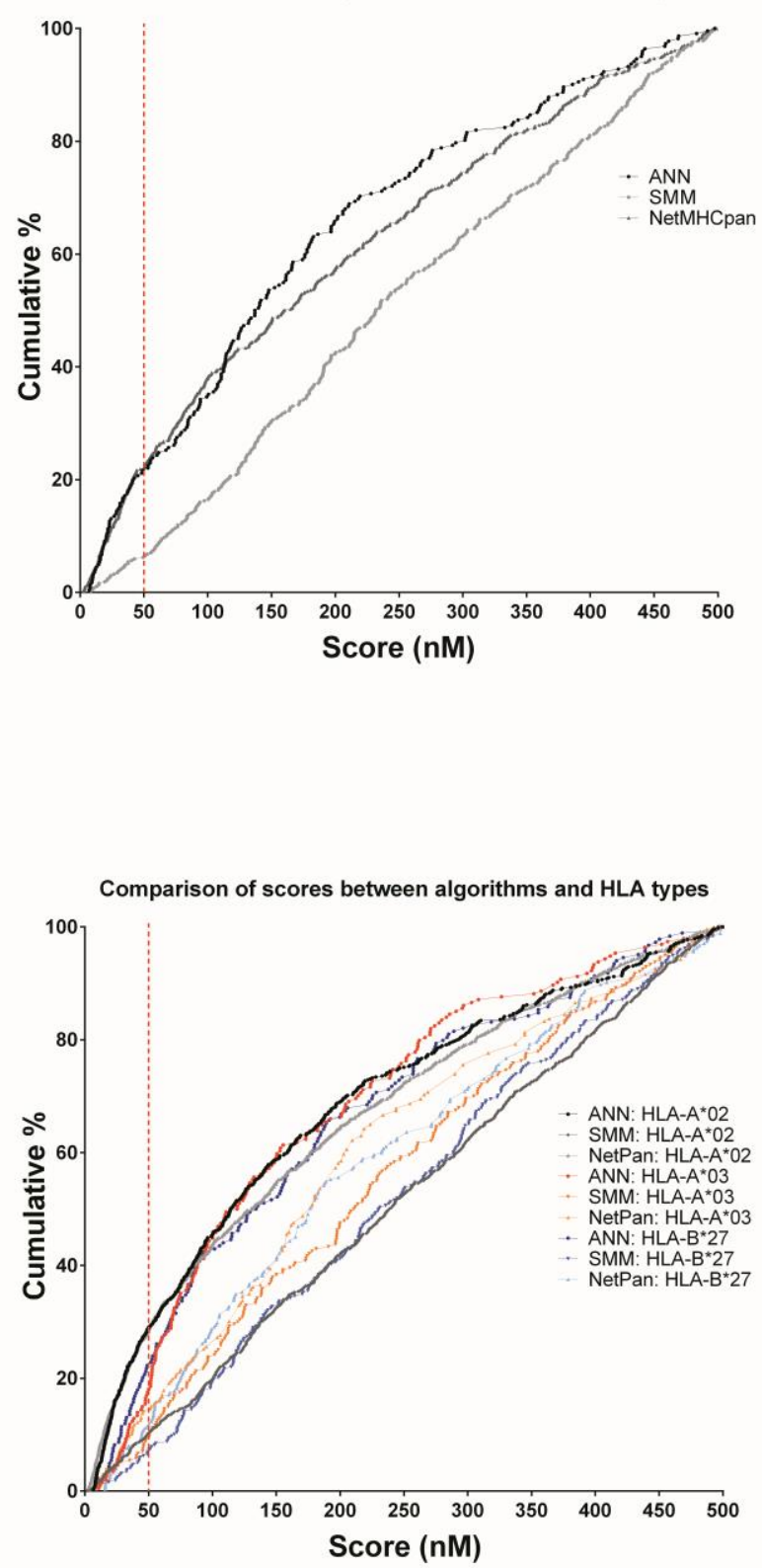

Downloaded from cancerimmunolres.aacrjournals.org on June 11, 2015. (c) 2015 American Association for Cancer Research. 
Figure 2
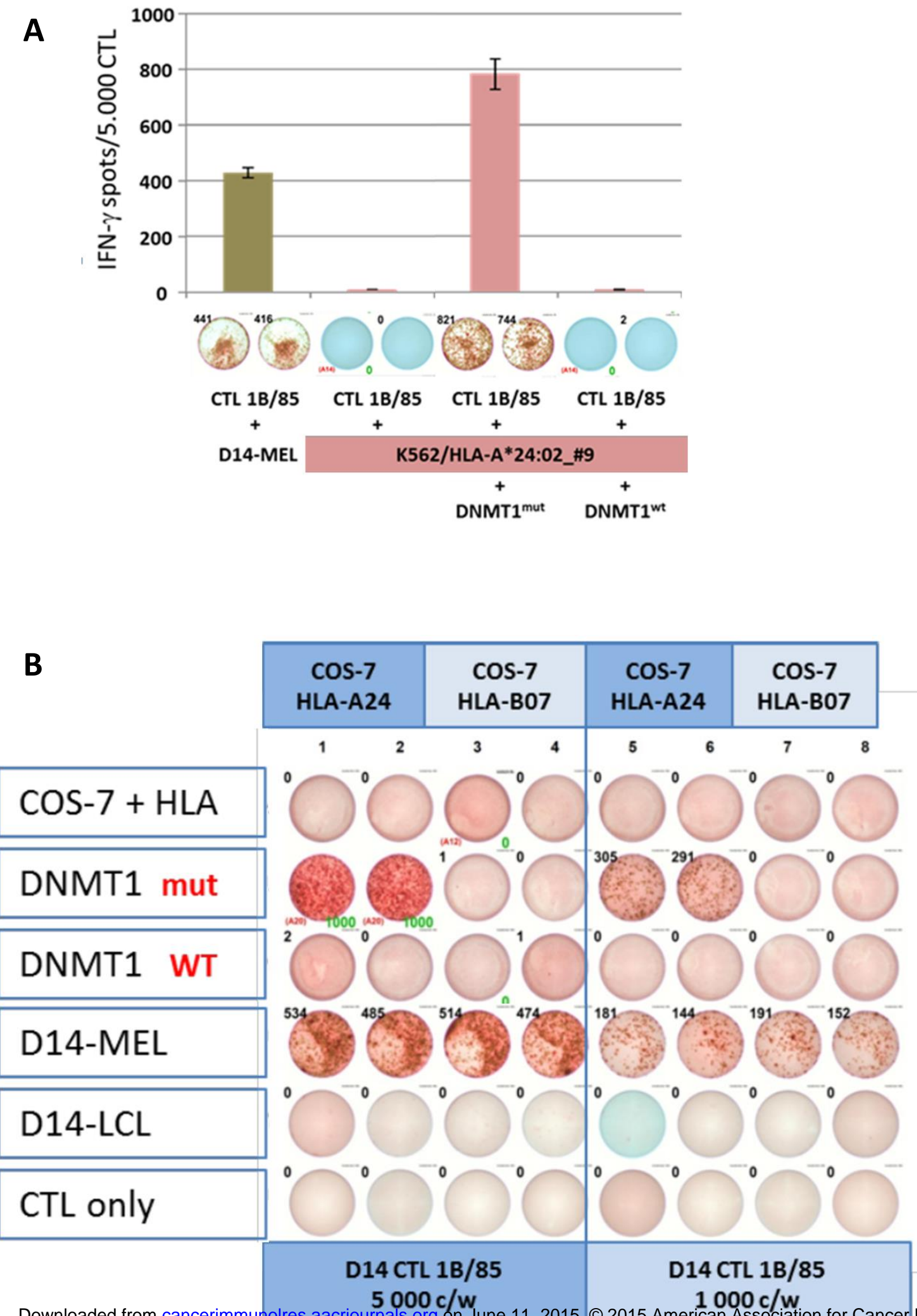


\section{AACR \\ Cancer Immunology Research}

\section{Exome sequencing to predict neoantigens in melanoma}

Antonia L Pritchard, Julie G Burel, Michelle A. Neller, et al.

Cancer Immunol Res Published OnlineFirst June 5, 2015.

Updated version Access the most recent version of this article at: doi:10.1158/2326-6066.CIR-15-0088

Supplementary Access the most recent supplemental material at:

Material http://cancerimmunolres.aacrjournals.org/content/suppl/2015/06/05/2326-6066.CIR-15-0088.DC1. $\mathrm{html}$

Author Author manuscripts have been peer reviewed and accepted for publication but have not yet been Manuscript edited.

\section{E-mail alerts Sign up to receive free email-alerts related to this article or journal.}

Reprints and To order reprints of this article or to subscribe to the journal, contact the AACR Publications

Subscriptions Department at pubs@aacr.org.

Permissions To request permission to re-use all or part of this article, contact the AACR Publications Department at permissions@aacr.org. 